setting, but this in no way detracts from the book, perhaps the only thing which does being the quality of schlieren photographs on which several of the papers depend. The photographs are in general poor, but there is no indication whether this is due to the printing process used or to the poor quality of the originals.

I. S. DONALDSON

\section{SURVEY OF ASTRONOMY}

Vistas in Astronomy

Edited by Arthur Beer. Vol. 3, Part 1: DynamicsGeophysics-Instruments-Solar System. Vol. 3, Part 2: Stellar Astronomy--Stellar Evolution-Photometry - Galaxies-Spectroscopy - Cosmology. (Special Supplement No. 7 to the Journal of Atmospheric and Terrestrial Physics.) Pp. vii +345. (London and New York: Pergamon Press, 1960.) 120s. net.

$\mathrm{T}$

HE third volume of Vistas in Astronomy, published, as in the case of the two preceding volumes, by Arthur Beer, opens with an article by R. van der R. Woolley, Astronomer Royal, on "The Dynamics of Stars in the Neighbourhood of the Sun". The author deals in particular with the movements of stars near at hand, the permanence of very open clusters and the equipartition of energy. R. A. Lyttleton poses the following question: "What is the simplest theoretical approach and minimum amount of calculation that could have led to the discovery of the planet Neptune ?" Tisserand had already posed this question in his Mécanique Céleste, when he suggested that it would suffice to assign successive values to the radius of the planet, which is supposed to have a circular orbit. Lyttleton shows that one can simplify the problem considerably without altering the numerical solution much, by attributing circular orbits to both Uranus and Neptune. The position which he finds for the hypothetical planet does not differ more than one degree from the observed position; as for its mass, he approximates the real value more closely than Adams and Le Verrier. But he has the adlantage over his two predecessors of knowing that Neptune exists, whereas in 1845 or 1846 it was doubtful, as the question posed by Airy to Adams at first and then to Le Verrier proves. What is more, we know that the orbit of Neptune has a slight eccentricity. The paths of discovery are often tortuous, but one does not perceive this until the goal has been attained.

The contribution of $\mathrm{H}$. von Klüber is also of great interest and of more immediate application. It is a discussion which takes into account all the detorminations, made since 1919, of the displacement of stars around the Sun when in eclipse. The existence of this effect is not in question, but the extent of displacement remains very uncertain. It appears, however, to be greater than that provided by theory. The observations are very scattered and, in conse. quence, the hyperbolical variation of the displacement as a function of the distance from the Sun cannot be considered as established. The recommendations directed by $\mathbf{H}$. von Klüber to future observers must be acclaimed without reserve.

Of interest are articles, one by W. Cochran, the other by V. Fock, on "The Clock Paradox"; some studies by G. M. Sisson on the "Design of Large Telescopes", by Marschal A. Wrubel on "The Elec. tronic Computer as an Astronomical Instrument", by A. H. Jarrett on "Techniques in Auroral Investigations" and by D. W. Beggs on "The Russian Satellites". Finally, four notes on the brilliant comets of 1957 complete the first part of this volume.

John B. Irwin opens the second part with an article entitled "The First 70,000". It is the exposition of a vast programme of observations destined to yield the following for all stars down to magnitude $8 \cdot 5$ : position, proper motion, magnitude, multicolours, spectral type, luminosity, parallax, radial velocity and polarization. The author emphasizes, by an apt title, the paucity of information we have on the southern hemisphere. He discusses the use of the transit circle for the determination of positions and proper motions, but he does not mention the use of the impersonal astrolabe for establishing the fundamental catalogue. Altogether, the programme outlined by John B. Irwin is excellent, but only international co-operation will allow it to be exocuted. It would be an interesting task for the International Astronomical Union.

Modern aspects of stellar photometry are dealt with in two articles, one by Gerald E. Kron on "Multiple Colour Photometry", the other by A. N. Argue entitled "Photometry with the Cambridge Schmidt Telescope". A long study by B. E.J. Pagel provides interesting ideas on "Tho Excitation of Emission Lines in the Spectra of Early-type Stars". Olin J. Eggen has prefaced his article, "Colour, Luminosity and Erolution of the Stars", with some opinions of this evolution by celebrated mon of science, from Helmholtz (1854) to Eddington (1926). This comparison of ideas put forward by very high authorities produces an effect of shock on the reader of to-day and inspires him to salutary reflexions on what H. Poincaré called "La Valeur de la Science". Eggen shows, with diverse examples, all that photometry of stellar clusters in several colours teaches us about stellar evolution. The volume ends with a representation of "The Visual Milky Way" by Sergei Gaposchkin and with two theoretical studies, one by L. Mestel entitled "Star Formation and the Galactic Magnetic Field" and another by D. W. Sciama called "Obserrational Aspects of Cosmology".

Presented with the samo care as the two preceding ones, Volume 3 of Vistas in Astronomy contains an abundant bibliography and a very detailed "Name and Subject Index"; it is an excellent piece of work. The editor announces the publication of two other volumes, the authors of which will be drawn from fifteen countries or so. Vistas in Astronomy is indeed of immense utility for all those who wish to know the state of advancement of the diverse branches of astronomy. A. Danson

\section{THE NATURAL HISTORY OF GALAXIES}

The Hubble At'as of Ga!axies

By Allan Sandage. Pp. viii $+32+50$ plates. (Washington, D.C. : Carnegie Institution of Washington, 1961.) 10 dollars.

THIS is a remarkable and fascinating book. Conceived by Edwin Hubble, who did not live to see it finished, and now completed by Hubble's pupil and successor, Dr. Allan Sandage, it can be recommended to a very wide range of readers, from 